\title{
Contrast and dyes
}

\section{NG Biyase}

Department of Anaesthesia, Charlotte Maxeke Johannesburg Academic Hospital and University of the Witwatersrand, South Africa Corresponding author, email: nana.biyase@yahoo.com

Keywords: contrast, dyes, imaging, anatomic boundaries

\section{Contrast}

Contrast agents have long been used for the imaging of anatomic boundaries and to explore normal and abnormal physiologic findings. The introduction of increasingly faster and more discriminating radiographic imaging techniques has resulted in the need for radiation-attenuating contrast agents that can be used in traditional radiographic imaging or, more recently, in subtraction imaging, both of which can be projected and rotated in three dimensions. By far the most successful and widely applied contrast agents in use today are the iodinated contrast agents (ICAs), first introduced into clinical practice in the 1950s. ${ }^{1}$ It is estimated that approximately 75 million doses of ICAs are given worldwide each year.

\section{Properties of the ideal contrast agent ${ }^{2}$}

- Non-toxic

- Should not cross blood-brain barrier
- Similar physiologic properties to blood

- Inertness

- Opacification

- Low surface tension

- Easy injectability

- Elimination

- Cost-effective

\section{Properties of iodinated contrast agents ${ }^{3-5}$}

The iodine molecule can absorb $\mathrm{x}$-rays to provide contrast and has been the basis of intravenous contrast since 1950s. All ICAs share a similar function group-a tri-iodinated benzene ring. The atomic radius of a covalently bonded iodine atom is approximately 133 picometers, which falls within the range of the wavelengths of $x$-rays: 10 to 10000 picometers; thus, $x$-rays are easily attenuated by the iodine atoms. Furthermore, three

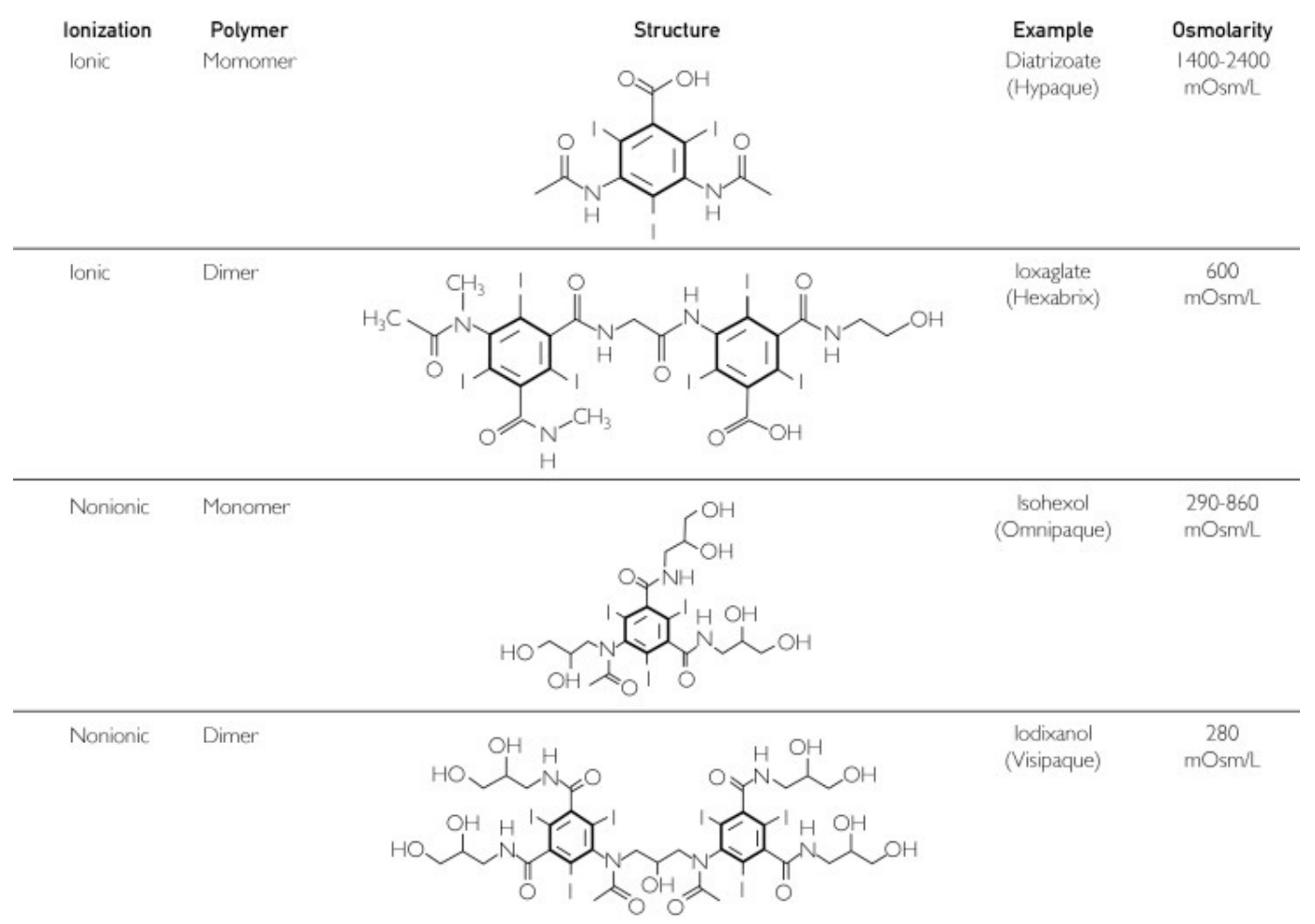

Figure 1: Properties of the four classes of iodinated contrast agents 
iodine atoms covalently bonded to a benzene ring offer two major advantages: (1) three large atoms located in such close proximity increase the effective molecular size, thus attenuating longer-wavelength $x$-rays, and (2) covalent bonding to a stable organic functional group (i.e. benzene) reduces the risk of toxic effects from free iodide.

Compounds consist of either one tri-iodinated benzene ring (i.e. monomers) or two tri-iodinated benzene rings linked by an organic functional group (i.e. dimers). In addition, ionic tendency is governed by the presence (i.e. ionic) or absence (i.e. non-ionic) of a carboxylate (-COO-) functional group contained on an organic side chain. Typically, because the carboxylate moiety adds a net negative charge to the molecule, these anionic agents are usually available as salts of sodium, calcium, or methylglucamine cations.

Hence, the four major classes of iodinated contrast agents are as follows (Figure 1):

1. lonic monomer: single tri-iodinated benzene ring with a carboxylate-containing benzene substituent.

2. Ionic dimer: two linked tri-iodinated benzene rings in which at least one carboxylate-containing group is substituted on at least one benzene ring.

3. Non-ionic monomer: single tri-iodinated benzene ring without a carboxylate-containing benzene substituent.

4. Non-ionic dimer: two linked tri-iodinated benzene rings that do not contain a carboxylate functional group within any benzene substituent.

lodinated contrast agents have several key properties that influence their behaviour, efficiency, and adverse reaction profile. Interplay among these properties optimises the degree of radio opacification and tolerability. These properties include:

Osmolality is a measure of the number of active particles when the agent is dissolved in $1 \mathrm{~kg}$ of water $(\mathrm{mOsm} / \mathrm{kg})$. lodinated contrast agents are classified as high-osmolality contrast media (HOCM), low-osmolality contrast media (LOCM), or iso-osmolar contrast media (IOCM). Older iodinated contrast agents are typically HOCM (1.300-2.140 mOsm/kg of water), whereas newer contrastagents developed from the 1980saretypicallyLOCM(600$850 \mathrm{mOsm} / \mathrm{kg}$ of water). IOCM iodinated contrast agents are a more recent development, with osmolality that equals that of blood. Contrast osmolality higher than blood (HOCM and LOCM) results in movement of water from interstitial spaces into the vascular compartment, which causes increased blood viscosity, endothelial damage, hypervolaemia, vasodilation, oedema with neurotoxicity, decreased myocardial contractility, and toxicity.

Viscosity of ICAs is determined by their flow friction, resistance, or thickness of the fluid. lodinated contrast agents are significantly more viscous than radiopharmaceuticals, and this attribute has an impact on intravenous injectability, flow rate, and gauge of cannula required. Viscosity can be reduced by injecting the agent at body temperature $\left(37^{\circ} \mathrm{C}\right)$ rather than room temperature. LOCM has higher viscosity than HOCM, and viscosity increases with iodine content. Viscosity plays an important role in renal tolerance, contributing significantly to the risk of contrastinduced nephrotoxicity.

lonicity is the tendency for the agent to separate into charged species (ions) when dissolved in solution. Ionic iodinated computed tomography (CT) contrast agents dissociate into ion pairs whereas non-ionic do not. Fully saturated tri-iodinated benzoic acid derivative monomer CT contrast agents dissociate into ions in solution, with the anion containing the iodine atoms and the cation containing sodium or meglumine. The dimer form (two tri-iodinated benzoic acid rings) allows high iodine content but low osmolality because a single cation is still all that is required. Only the anion is radiopaque, because it carries the iodine atoms, but the cation is needed for the solution to be formed.

lodine concentration among iodinated $\mathrm{CT}$ contrast agents ranges from 11-46\%, with higher iodine concentrations providing both better radio-opacification and a higher risk of adverse reactions.

\section{Mechanism of action ${ }^{5}$}

The $\mathrm{K}$ edge refers to the abrupt increase in attenuation when the energy of the incident $\mathrm{x}$-ray approximates the $\mathrm{K}$ binding energy. The human body is largely composed of low-atomic-mass elements with corresponding low-K shell binding energies. Higher atomic masses associated with, among many elements, iodine, gadolinium, and lead have high-K shell binding energies producing characteristic $x$-rays with relatively high energies. This principle allows higher-atomic-mass elements to be used for contrast media or detector material. Since the photoelectric effect has a higher probability of occurring between low-energy $\mathrm{x}$-rays and high-atomic-mass elements, it provides excellent properties as a CT contrast agent (Figure 2).

\section{Pharmacokinetics}

lodinated CT contrast agents are best described using a 2-compartment model. lodinated contrast agents demonstrate a rapid peak plasma concentration at $2 \mathrm{~min}$ after intravenous

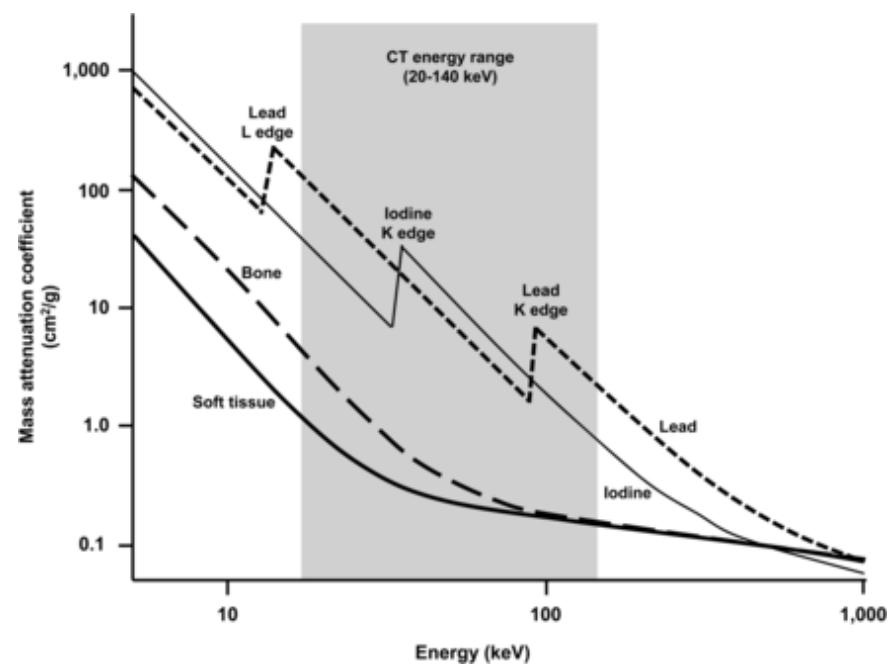

Figure 2: Mass attenuation coefficients against x-ray energy 
injection. A biphasic plasma profile represents rapid diffusion of the contrast from the plasma compartment into the interstitial compartment, and then slows urinary clearance. The peak plasma concentration at $2 \mathrm{~min}$ after intravenous injection shortens to 1 min with higher doses. Only $1-3 \%$ of iodinated contrast agents undergo plasma protein-binding. As contrast diffuses into the extravascular space, water is drawn from the extravascular space into the intravascular space because of osmolality. Tight junctions prevent movement of contrast into extravascular spaces in the brain, testes, and neural tissues.

The biphasic half-lives vary from one agent to the next but tend to be approximately 7 minutes (range 2-30 minutes) and 1.6 hours (range 1-2 hours), with these half-lives universally increasing with increasing contrast dose. In renal dysfunction, elimination half-life can increase to 40 hours. The intravenous CT contrast agents do not undergo metabolism and are excreted almost exclusively by glomerular filtration in the urine unchanged. Calcium or magnesium has been added to the newer longer contrast agents to reduce toxicity. All ionic contrast media bind in vivo to calcium and magnesium. If given orally, CT contrast agents are absorbed from the intestine, glucuroconjugated, strongly plasma protein-bound, and then rapidly concentrated and eliminated in the biliary system.

\section{Adverse reactions $^{5-7}$}

Acute adverse reactions to ICAs occur within one hour of use. The overall incidence of severe acute reactions to ICAs depends on the class of agent administered.

Specifically, severe acute reactions are five times more common after the administration of an ionic, monomeric, high-osmolality agent vs a low- or iso-osmolar agent with a risk of $0.22 \%$ vs $0.04 \%$. Overall mortality from acute reactions to ICAs is 1:13 000

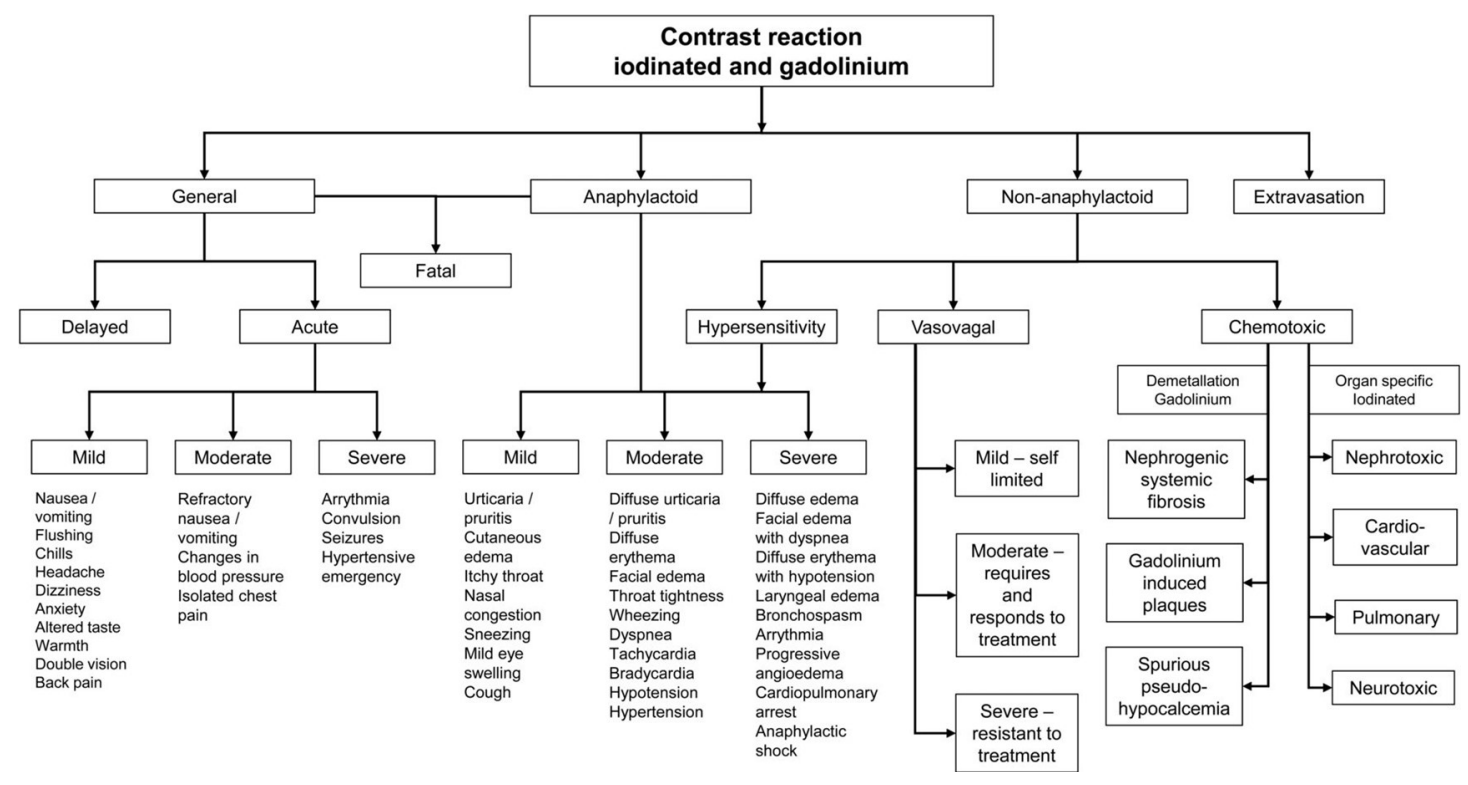

Figure 3: Classification of contrast reactions

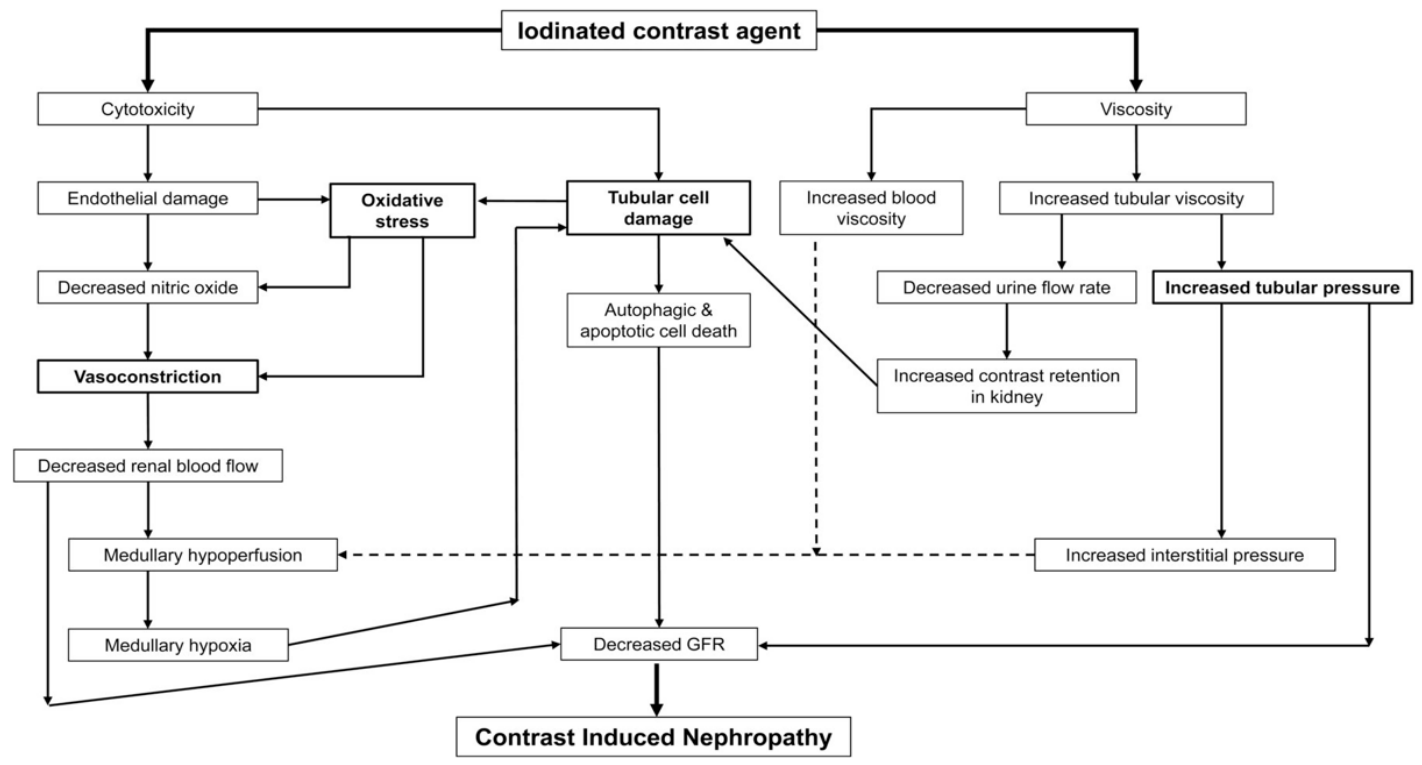

Figure 4: Pathophysiology of contrast-induced nephropathy 
to $1: 169000$. The severity of these reactions can range from mild to severe and life-threatening (Figure 3).

Contrast-induced nephropathy (CIN) is one of the most explored adverse effect; it refers to a reduction in renal function after the administration of an ICA. The standard diagnostic criteria for $\mathrm{CIN}$ is a greater than $25 \%$ increase in baseline serum creatinine concentration within three days of receiving an ICA after other possible causes have been ruled out. Serum creatinine will usually peak within 3-7 days and return to baseline (or a new baseline) within 14 days. In many patients, the course is usually benign; however, the development of CIN can prolong hospital stay, increase the need for dialysis, and increase overall mortality. Figure 4 outlines the pathophysiology of CIN and the management thereof will not be discussed as it is beyond the scope of this lecture.

Adverse reactions to ICAs are common; however, severe reactions are rare. Most severe acute reactions and $\mathrm{CIN}$ occur with high-osmolality ionic monomeric ICAs. Delayed reactions are most common after non-ionic dimers. Identification of patients who are at risk for adverse reactions and minimising risk by following preventive measures or using alternative (i.e. ICA independent) diagnostic techniques can be helpful in reducing the incidence of adverse effects.

Dyes

Fluorescence imaging ( $\mathrm{Fl})$ is one of the most popular imaging modes in biomedical sciences for the visualisation of cells and tissues both in vitro and in vivo. The benefits of $\mathrm{Fl}$ include: ${ }^{8}$

- High contrast, that is, signal to noise ratio (SNR): only the target, not background, is visible because separate wavelengths are used for illumination and recording.

- High sensitivity: extremely small concentrations can often be made visible.

- Gives molecular information: makes some (bio) chemistry spatially and temporally visible.

- Cheap: the optical instrumentation and computing needed are quite simple.

- Easy to use: resembles classical staining.

While near-infrared fluorescence (NIRF) imaging has recognised potential, only indocyanine green (ICG) is FDA approved for clinical use. ${ }^{9}$ Indocyanine green dye was developed for nearinfrared (NIR) photography by the Kodak Research Laboratories in 1955 and was approved for clinical use already in 1956. However, it took over ten years before ICG was used for angiography. For retinal angiography it has been used from the early 1970 s.

\section{Properties of indocyanine green $^{8}$}

ICG has several clinically excellent properties, which have been thoroughly verified during its long clinical use:

- Patient safety: non-toxic and non-ionising.

- Ideal for angiography: binds efficiently to blood lipoproteins, that is, it does not leak from circulation.
- Short life time in blood circulation allowing repeated applications.

- Good SNR: there is not much NIR autofluorescence in tissue giving low noise background.

- Deep imaging: operates in tissue optical window (NIR).

- Simple and cheap imaging devices.

Indocyanine green is a tricarbocyanine dye having a molecular weight of 751.4 Da. It is a negatively charged ion that belongs to the large family of cyanine dyes. Dry ICG is stable at room temperature. This is also the form of pharmaceutically available ICG. ICG is soluble in water $(1 \mathrm{mg} / \mathrm{ml})$ but is not readily soluble in saline. Some chemicals, such as sodium polyaspartate (PASP), can be used to stabilise ICG in water and blood solutions, for example, when blood samples should be stored for several days. Also, storage of the ICG solution at low temperature $\left(4{ }^{\circ} \mathrm{C}\right)$ inhibits decomposition, while storage at room temperature facilitates decomposition.

The spectral stabilisation is fastest when ICG is dissolved in distilled water, and thus it is not recommended to add isotonic saline and/or albumin to the injectate when fast spectral stability is essential, for example, when using ICG for quantitative purposes.

The important property of fast binding to plasma proteins, especially lipoproteins, makes repeated intraoperational applications of ICG possible. The binding to plasma proteins does not seem to alter protein structures, which is one sign of non-toxicity. It seems that ICG actually binds to the lipids of lipoprotein complexes ( $\beta$-lipoprotein), and that the bind results in more intense fluorescence than ICG bound to, for example, free cholesterol. Binding to blood proteins also shifts slowly, taking several minutes, the absorption peak at $780 \mathrm{~nm}$ towards longer wavelengths to $805 \mathrm{~nm}$.

\section{Pharmacokinetics}

ICG does not have any known metabolites, and it is rapidly extracted by the liver into bile secretions. A protein called glutathione S-transferase does the transportation without modification. Caloric restriction seems to significantly increase the plasma clearance rate at low doses $(0.5 \mathrm{mg} / \mathrm{kg})$. The protein spectra of different liver diseases also affect ICG protein binding in blood.

The typical dye concentrations used for in vivo retinal and choroidal angiography are in the range of $20-25 \mathrm{mg} / \mathrm{ml}$ of ICG applied by injection into a peripheral arm vein. For studies of hepatic function an intravenous injection dose is calculated on the basis of $0.5 \mathrm{mg} / \mathrm{kg}$ of body weight. In cardiac output and blood volume monitoring the total dose of dye injected should be kept below $2 \mathrm{mg} / \mathrm{kg}$. No significant toxic effects have been observed in humans with the high dose of $5 \mathrm{mg} / \mathrm{kg}$ of body weight. 


\section{Penetration $^{8,10}$}

ICG works in the so-called tissue optical window, that is, the NIR light used both in excitation and fluorescence penetrates tissue several millimetres or even further. This translucency helps to observe, for example, vascular structures that might be buried in clots or dura. The penetration depth of light energy into skin and underlying tissues can be calculated on the basis of in vivo measurements of optical density (OD) (accounting scattering and absorption) of the human skin and underlying tissues defined as $O D=\log _{10} I_{0} / I_{t}$, where $I_{0}$ is the measured back reflected intensity, and $l_{t}$ is that of the reference.

\section{Clinical applications ${ }^{8}$}

Neurosurgery: In revascularisation, that is, bypass procedures, the patency of the vascular microanastomosis is likewise paramount to successful procedures. Incomplete obliteration of a rupture-prone aneurysm or arteriovenous malformation (AVM) may result in a haemorrhage, and occlusion of a parent vessel or an anastomosis in an ischaemic stroke; both of which may have catastrophic consequences for the patient. Postoperative angiography is useful in assessing the residual filling of the treated lesion, but in case of inadvertent vessel occlusion the result of postoperative imaging comes too late, and the ischaemic brain or medullary lesion has already irreversibly occurred.

Coronary surgery: NIR based on the intravascular ICG dosing has emerged as a novel method for graft patency assessment. Two main methods are used, firstly an indirect method in which the myocardial tissue perfusion is assessed by imaging an area of interest around a coronary vessel; secondly, a direct imaging of the grafts by visualising the graft lumen by ICG angiography.

Reconstructive microsurgery: In perforator flaps, the perfusion of the most distal parts of the flaps is often problematic. Recently, several reports have shown the feasibility of ICG angiography in the intraoperative assessment of flap viability. ICG angiography has also been used to assess the patency of the microvascular anastomosis intraoperatively, and the intrinsic transit time of the flap circulation with promising results. In the preoperative planning of perforator flap reconstructions, ICG angiography has been used to detect suitable perforator vessels, which facilitates the design of the flap. After free tissue transfer surgery, the flap circulation has to be carefully followed. Although other methods exist for continuous flap monitoring, ICG angiography may be helpful in the early postoperative phase for the detection of anastomotic thrombosis, when flap survival is in doubt.

\section{Conclusion}

Radiologists have to choose a particular contrast agent knowing its physical and chemical properties and the possibility of adverse reaction and balancing them with the clinical benefits of a more precise diagnosis.

\section{Conflict of interest}

The author declares no conflict of interest.

\section{Funding source}

None.

\section{ORCID}

NG Biyase (iD https://orcid.org/0000-0002-2120-0162

\section{References}

1. Kinch MS, Woodard PK. Analysis of FDA-approved imaging agents. Drug Discov Today. 2017;22(7):1077-83. https://doi.org/10.1016/j.drudis.2017.03.006.

2. Jascinth A, Reddy C, Mouli G, et al. Contrast agents in computed tomography: $A$ review. Journal of Applied Dental and Medical Sciences. 2016;2(2):143-9.

3. Bourin $M$, Jolliet $P$, Ballereau, $F$. An overview of the clinical pharmacokinetics of X-ray contrast media. Clin-Pharmacokinet. 1997;2:180-93. https://doi. org/10.2165/00003088-199732030-00002.

4. Cascher L, Lazzara A, Piergallini L, et al. Contrast agents in diagnostic imaging Present and future. Pharmacol Res. 2016;110:65-75. https://doi.org/10.1016/j. phrs.2016.04.023.

5. Currie GM. Pharmacology, Part 5: CT and MRI Contrast Media. J Nucl Med Technol. 2019;47(3):189-202. https://doi.org/10.2967/jnmt.118.220012.

6. Beckett K, Moriarity A, Langer J. Safe use of contrast media: what the radiologist needs to know. Radiographics 2015;35(6):1738-50. https://doi.org/10.1148/ rg.2015150033.

7. Thomsen HS, Webb JAW, editors. Contrast media. Safety issues and ESUR guidelines. 3rd ed. Heidelberg: Springer; 2013.

8. Alander J, Kaartinen I, Laakso A, et al. A review of indocyanine green fluorescent imaging in surgery. Int J Biomed Imaging 2012;2012:940585. https://doi. org/10.1155/2012/940585.

9. Pansare V, Hejazi S, Faenza W, Prud'homme R. Review of long-wavelength optical and NIR imaging materials: contrast agents, fluorophores, and multifunctional nano carriers. Chem Mater. 2012;24(5):812-27. https://doi.org/10.1021/ cm2028367.

10. Marshall M, Rasmussen J, Tan I, et al. Near-infrared fluorescence imaging in humans with indocyanine green: a review and update. Open Surg Oncol J. 2010;2(2):12-25. https://doi.org/10.2174/1876504101002010012. 